\title{
Invertibility of Operators in Spaces of Real Interpolation
}

\author{
Irina ASEKRITOVA and Natan KRUGLYAK \\ The School of Mathematics and Systems Engineering \\ Department of Mathematics \\ Växjö University \\ SE-351 95, Växjö - Sweden \\ Luleå University of Technology \\ SE-971 87, Luleå — Sweden \\ natan@ltu.se
}

Received: August 18, 2007

Accepted: September 17, 2007

\begin{abstract}
Let $A$ be a linear bounded operator from a couple $\vec{X}=\left(X_{0}, X_{1}\right)$ to a couple $\vec{Y}=\left(Y_{0}, Y_{1}\right)$ such that the restrictions of $A$ on the spaces $X_{0}$ and $X_{1}$ have bounded inverses. This condition does not imply that the restriction of $A$ on the real interpolation space $\left(X_{0}, X_{1}\right)_{\theta, q}$ has a bounded inverse for all values of the parameters $\theta$ and $q$. In this paper under some conditions on the kernel of $A$ we describe all spaces $\left(X_{0}, X_{1}\right)_{\theta, q}$ such that the operator $A:\left(x_{0}, X_{1}\right)_{\theta, q} \rightarrow\left(Y_{0}, Y_{1}\right)$ has a bounded inverse.
\end{abstract}

Key words: real interpolation, invertible operators.

2000 Mathematics Subject Classification: Primary 46B70, Secondary 46M35.

\section{Introduction}

In the area of partial differential equations, the importance of invertibility of operators in scales of spaces was first observed by Alberto Calderón in 1985 [5], who considered the case of $L^{p}$ scale and an operator bounded in $L^{2}$. New applications of invertibility of operators to PDE were recently obtained by Kalton and Mitrea [10]. These applications are closely connected to interpolation theory and, in particular, to the remarkable theorem proved by I. Ya. Shneiberg (see $[16,17])$. This theorem in its simplest form claims that if a linear bounded operator $A$ from a couple $\vec{X}=\left(X_{0}, X_{1}\right)$ to itself is invertible on a complex interpolation space $\left[X_{0}, X_{1}\right]_{\theta_{0}}$, then it is also invertible on the spaces $\left[X_{0}, X_{1}\right]_{\theta}$ when $\theta$ is close to $\theta_{0}:\left|\theta-\theta_{0}\right|<\varepsilon$. Later on different 
generalizations and applications of Shneiberg's results were obtained by various authors (see, for example, $[2,6,7,11,19,20]$ ). In particular, in the work [11] a general theory of Shneiberg-type theorems was proposed.

The above mentioned applications are closely connected to the following problem. Let $A$ be a linear bounded operator from a Banach couple $\vec{X}=\left(X_{0}, X_{1}\right)$ to a Banach couple $\vec{Y}=\left(Y_{0}, Y_{1}\right)$. Let also $\Omega_{q}$ be the set of all $\theta$ for which the restriction of the operator $A$ on the space $\left(X_{0}, X_{1}\right)_{\theta, q}$ has a bounded inverse defined on the space $\left(Y_{0}, Y_{1}\right)_{\theta, q}$. Then it follows from an analog of Shneiberg theorem (proved for the case $q<\infty$ in [20] and proved for the general case, including $q=\infty$, in [11]) that the set $\Omega_{q}$ is open. To describe the set $\Omega_{q}$, the following problem has to be solved:

Problem. Suppose that the restrictions of the operator $A$ on the spaces $X_{0}$ and $X_{1}$ have bounded inverses defined on the spaces $Y_{0}$ and $Y_{1}$, respectively. How can we describe all real interpolation spaces $\left(X_{0}, X_{1}\right)_{\theta, q}$ such that the restriction of the operator $A$ on a space $\left(X_{0}, X_{1}\right)_{\theta, q}$ has a bounded inverse on the space $\left(Y_{0}, Y_{1}\right)_{\theta, q}$ ?

Two different but complimentary approaches to this problem are possible. The first approach consists of a complete and, if possible, explicit description of the set $\Omega_{q}$. In the general case, this task is rather complicated, even in the case when the kernel of the operator $A$ is of dimension one. Let us also note that the proofs known for this case are based on Hahn-Banach theorem and are not constructive (see $[1,9]$ ).

The second approach consists of finding sufficiently simple and easily tested conditions that would allow for a complete solution of the problem. A constructive solution is preferable since the problem can, in fact, be reduced to the problem of solving the equation

$$
A x=y,
$$

where $y \in\left(Y_{0}, Y_{1}\right)_{\theta, q}$ and $\theta$ does not belong to the set $\Omega_{q}$.

The present work takes the first step in developing the second approach. Our main result is the following

Theorem A. Let $A$ be a bounded linear operator from a Banach couple $\vec{X}=\left(X_{0}, X_{1}\right)$ to a Banach couple $\vec{Y}=\left(Y_{0}, Y_{1}\right)$ such that $A$ is invertible on the spaces $X_{0}$ and $X_{1}$. Suppose also that its kernel $\operatorname{Ker} A \subset X_{0}+X_{1}$ is finite-dimensional and has a basis $e_{1}, \ldots, e_{n}$ such that

$$
K\left(t, e_{i} ; X_{0}, X_{1}\right) \approx t^{\theta_{i}} \quad\left(\theta_{i} \in(0,1), \theta_{i} \neq \theta_{j} \quad \text { for } i \neq j\right) .
$$

Then the operator $A$ is invertible on the space $\left(X_{0}, X_{1}\right)_{\theta, q}$ if and only if $\theta \neq \theta_{i}$ $(i=1, \ldots, n)$.

A direct constructive proof of this result will be presented below. It is easy to see, especially in the case when the kernel is one-dimensional, how the algorithm for constructing the solution to the equation $A x=y, y \in\left(Y_{0}, Y_{1}\right)_{\theta, q}$, changes as the parameter $\theta$ passes a critical value $\theta_{i}$. 
The following example, taken from [12], illustrates this theorem. Let $L_{1}\left(t^{-\alpha}, \frac{d t}{t}\right)$ be a space of functions on $(0, \infty)$ defined by the norm

$$
\|f\|_{L_{1}\left(t^{-\alpha}, \frac{d t}{t}\right)}=\int_{0}^{\infty}|f(t)| t^{-\alpha} \frac{d t}{t}<\infty
$$

and let us consider an operator $A=I-H$ (Identity minus Hardy) which is defined by the formula $(A f)(t)=f(t)-\frac{1}{t} \int_{0}^{t} f(s) d s$. Let also $\left(X_{0}, X_{1}\right)=\left(L_{1}\left(\sqrt{t}, \frac{d t}{t}\right), L_{1}\left(\frac{1}{\sqrt{t}}, \frac{d t}{t}\right)\right)$. It is easy to verify that the operator $A=I-H$ has a one-dimensional kernel in $X_{0}+X_{1}$ which consists of constant functions $f(x) \equiv C$. Note that for $f(x) \equiv C$ holds

$$
K\left(t, f ; X_{0}, X_{1}\right)=\int_{0}^{\infty} C \min \left(\sqrt{s}, \frac{t}{\sqrt{s}}\right) \frac{d s}{s} \approx C \sqrt{t} .
$$

As the operator $A$ is bounded and invertible on the spaces $X_{0}$ and $X_{1}$ (see [12]), therefore the conditions of Theorem A are fulfilled. Hence Theorem A describes all spaces $\left(X_{0}, X_{1}\right)_{\theta, q}$ on which $A=I-H$ is invertible.

We will prove the theorem in two steps. In the first step we reduce the theorem to the case when the kernel of the operator $A$ is one-dimensional and in the second step we consider the case of a one-dimensional kernel.

\section{Reduction to the case of a one-dimensional kernel}

First of all let us note that it is sufficient to consider the case when $A$ is a quotient operator. Indeed, if we denote by $\vec{A}: \vec{X} \rightarrow \vec{X} / \operatorname{Ker} A$ the quotient operator then we have $A=B \bar{A}$, where $B: \vec{X} / \operatorname{Ker} A \rightarrow \vec{Y}$ is invertible on the end spaces and has no kernel. Therefore, $B$ is an invertible operator for all interpolation spaces $\left(X_{0}, X_{1}\right)_{\theta, q}$, and it is sufficient to prove the theorem for the operator $\bar{A}$. Note that $\bar{A}$ can be represented as a product $\bar{A}=A_{n} A_{n-1} \cdots A_{1}$, where $A_{1}$ is an operator with the kernel Ker $A_{1}=\operatorname{Span}\left\{e_{1}\right\}$ and $A_{i}(i=2, \ldots, n)$ is an operator with a one-dimensional kernel generated by the element $A_{i-1} \cdots A_{1} e_{i}$. Therefore, Theorem A can be easily proved by induction using the following result.

Theorem 1.1. If an operator A from a couple $\vec{X}$ to a couple $\vec{Y}$ is invertible on the spaces $X_{0}$ and $X_{1}$ and has a one-dimensional kernel $\operatorname{Ker} A=\{\lambda e\}$ such that $K(t, e ; \vec{X}) \approx t^{\theta_{0}}$, then from $K(t, x ; \vec{X}) \approx t^{\theta}$ with $\theta \neq \theta_{0}$ it follows that

$$
K(t, A x ; \vec{Y}) \approx t^{\theta} .
$$

The proof of the theorem is based on the following lemma.

Lemma 1.2. Suppose that the operator $A: \vec{X} \rightarrow \vec{Y}$ is such that $A\left(X_{i}\right)=Y_{i}$ $(i=0,1)$. Then for any $x \in X_{0}+X_{1}$ holds

$$
K(t, A x ; \vec{Y}) \approx \inf _{u \in \operatorname{Ker} A} K(t, x-u ; \vec{X})
$$

with the constant of equivalence independent of $x$ and $t$. 
Proof. Let $u \in \operatorname{Ker} A$ and let $x_{0} \in X_{0}$ and $x_{1} \in X_{1}$ be some decomposition of $x-u$, i.e., $x-u=x_{0}+x_{1}$. Then

$$
A x=A x_{0}+A x_{1}
$$

and

$$
K(t, A x ; \vec{Y}) \leq\left\|A x_{0}\right\|_{Y_{0}}+t\left\|A x_{1}\right\|_{Y_{1}} \leq\|A\|\left(\left\|x_{0}\right\|_{X_{0}}+t\left\|x_{1}\right\|_{X_{1}}\right) .
$$

Hence

$$
K(t, A x ; \vec{Y}) \leq\|A\| \inf _{u \in \operatorname{Ker} A} K(t, x-u ; \vec{X}) .
$$

To prove the opposite inequality let us consider a decomposition $A x=y_{0}+y_{1}$ with $y_{0} \in Y_{0}$ and $y_{1} \in Y_{1}$. Since $A\left(X_{i}\right)=Y_{i}(i=0,1)$ we can find such elements $x_{0} \in X_{0}$ and $x_{1} \in X_{1}$ that $A x_{i}=y_{i}(i=0,1)$ and $\left\|x_{i}\right\|_{X_{i}} \leq c\left\|y_{i}\right\|_{Y_{i}}(i=0,1)$ with the constant $c>0$ independent of $y_{0}, y_{1}$, and $x$. Then from the equality

$$
A x=y_{0}+y_{1}=A x_{0}+A x_{1}
$$

it follows that $x-x_{0}-x_{1}=u \in \operatorname{Ker} A$ and

$$
K(t, x-u ; \vec{X}) \leq\left\|x_{0}\right\|_{X_{0}}+t\left\|x_{1}\right\|_{X_{1}} \leq c\left(\left\|y_{0}\right\|_{Y_{0}}+t\left\|y_{1}\right\|_{Y_{1}}\right) .
$$

Hence

$$
\inf _{u \in \operatorname{Ker} A} K(t, x-u ; \vec{X}) \leq c K(t, A x ; \vec{Y}) .
$$

Let us now return to the proof of Theorem 1.1.

Proof. From Lemma 1.2 it follows that it is sufficient to prove that the conditions

$$
\begin{aligned}
c_{0} t^{\theta_{0}} & \leq K(t, e ; \vec{X}) \leq c_{1} t^{\theta_{0}}, \\
d_{0} t^{\theta} & \leq K(t, x ; \vec{X}) \leq d_{1} t^{\theta}
\end{aligned}
$$

imply

$$
\inf _{\lambda} K(t, x-\lambda e ; \vec{X}) \approx t^{\theta} .
$$

Here $c_{0}, c_{1}, d_{0}$, and $d_{1}$ are some positive constants.

As

$$
K(t, A x, \vec{Y}) \approx \inf _{\lambda} K(t, x-\lambda e ; \vec{X}) \leq K(t, x ; \vec{X}) \leq d_{1} t^{\theta}
$$

it is sufficient to prove the estimate from below

$$
\inf _{\lambda} K(t, x-\lambda e ; \vec{X}) \geq \delta t^{\theta} .
$$

Let us fix a number $t>0$. From the inequality

$$
K(t, x-\lambda e ; \vec{X}) \geq K(t, \lambda e ; \vec{X})-K(t, x ; \vec{X}) \geq|\lambda| c_{0} t^{\theta_{0}}-d_{1} t^{\theta}
$$


it follows that if

$$
|\lambda| \geq \frac{2 d_{1}}{c_{0} t^{\theta_{0}-\theta}}
$$

then $K(t, x-\lambda e ; \vec{X}) \geq d_{1} t^{\theta}$ and it is sufficient to consider the case when

$$
|\lambda|<\frac{2 d_{1}}{c_{0} t^{\theta_{0}-\theta}} .
$$

Now we will consider the two cases $\theta>\theta_{0}$ and $\theta<\theta_{0}$ separately. In the case of $\theta>\theta_{0}$ from the concavity of the $K$-functional it follows that for any $T \geq t$ we have

$$
\begin{aligned}
K(t, x-\lambda e ; \vec{X}) & \geq \frac{t}{T} K(T, x-\lambda e ; \vec{X}) \geq \frac{t}{T}(K(T, x ; \vec{X})-|\lambda| K(T, e ; \vec{X})) \\
& \geq \frac{t}{T}\left(d_{0} T^{\theta}-\frac{2 d_{1}}{c_{0} t^{\theta_{0}-\theta}} c_{1} T^{\theta_{0}}\right) .
\end{aligned}
$$

If $T=\gamma t(\gamma>1)$ then

$$
K(t, x-\lambda e ; \vec{X}) \geq \frac{1}{\gamma}\left(d_{0} \gamma^{\theta} t^{\theta}-\frac{2 d_{1}}{c_{0} t^{\theta_{0}-\theta}} c_{1} \gamma^{\theta_{0}} t^{\theta_{0}}\right) .
$$

Let now $\gamma$ be such that

$$
d_{0} \gamma^{\theta}=\frac{3 d_{1}}{c_{0}} c_{1} \gamma^{\theta_{0}}
$$

Since $\theta>\theta_{0}, d_{1} \geq d_{0}$, and $c_{1} \geq c_{0}$, therefore $\gamma>1$ and we have

$$
K(t, x-\lambda e ; \vec{X}) \geq\left(\frac{1}{\gamma} \frac{d_{1}}{c_{0}} c_{1} \gamma^{\theta_{0}}\right) t^{\theta}=\delta t^{\theta},
$$

with the constant $\delta>0$ dependent only on the constants $\theta, \theta_{0}, d_{1}, d_{0}, c_{1}$, and $c_{0}$. In the case of $\theta<\theta_{0}$ we take $T=\gamma t$ with $\gamma<1$. From the properties of the $K$-functional we obtain the inequalities

$$
\begin{aligned}
K(t, x-\lambda e ; \vec{X}) & \geq K(T, x-\lambda e ; \vec{X}) \geq K(T, x ; \vec{X})-|\lambda| K(T, e ; \vec{X}) \\
& \geq d_{0} T^{\theta}-\frac{2 d_{1}}{c_{0} t^{\theta_{0}-\theta}} c_{1} T^{\theta_{0}}=t^{\theta}\left(d_{0} \gamma^{\theta}-\frac{2 d_{1}}{c_{0}} c_{1} \gamma^{\theta_{0}}\right) .
\end{aligned}
$$

Since $\theta<\theta_{0}$ we can choose such $\gamma<1$ that

$$
d_{0} \gamma^{\theta}=\frac{3 d_{1}}{c_{0}} c_{1} \gamma^{\theta_{0}}
$$

For such $\gamma$ we have

$$
K(t, x-\lambda e ; \vec{X}) \geq \frac{d_{1}}{c_{0}} c_{1} \gamma^{\theta_{0}} t^{\theta}=\delta t^{\theta},
$$

with the constant $\delta>0$ dependent only on the constants $\theta, \theta_{0}, d_{1}, d_{0}, c_{1}$, and $c_{0}$. 


\section{The case of a one-dimensional kernel}

Let $A: \vec{X} \rightarrow \vec{Y}$ be a bounded linear operator which is invertible on spaces $X_{0}$ and $X_{1}$. Suppose also that $A$ has in $X_{0}+X_{1}$ a one-dimensional kernel $\operatorname{Ker} A=\{\lambda e\}$ with $K(t, e ; \vec{X}) \approx t^{\theta_{0}}$. We need to prove that $A$ is invertible on the space $\left(X_{0}, X_{1}\right)_{\theta, q}$ if and only if $\theta \neq \theta_{0}$.

We start with the case when $\theta \neq \theta_{0}$. Since $K(t, e ; \vec{X}) \approx t^{\theta_{0}}$, therefore $\operatorname{Ker} A \cap$ $\left(X_{0}, X_{1}\right)_{\theta, q}=\{0\}$ and it is sufficient to show that for a given $y \in\left(Y_{0}, Y_{1}\right)_{\theta, q}$ it is possible to construct an element $x \in\left(X_{0}, X_{1}\right)_{\theta, q}$ such that $A x=y$ and $\|x\|_{\left(X_{0}, X_{1}\right)_{\theta, q}} \leq$ $\gamma\|y\|_{\left(Y_{0}, Y_{1}\right)_{\theta, q}}$ with $\gamma$ independent of $y$. From the equivalence theorem of the $K$ - and $J$-methods (see [4]) it follows that there exists a sequence of elements $y_{n} \in Y_{0} \cap Y_{1}$, $n \in \mathbb{Z}$, such that

$$
\left(\sum_{n \in \mathbb{Z}}\left(2^{-\theta n} J\left(2^{n}, y_{n} ; \vec{Y}\right)\right)^{q}\right)^{\frac{1}{q}} \leq \gamma\|y\|_{\left(Y_{0}, Y_{1}\right)_{\theta, q}},
$$

where $J\left(2^{n}, y_{n} ; \vec{Y}\right)=\max \left\{\left\|y_{n}\right\|_{Y_{0}}, 2^{n}\left\|y_{n}\right\|_{Y_{1}}\right\}$. As the operator $A$ has inverses on the spaces $X_{0}$ and $X_{1}$ defined on the spaces $Y_{0}$ and $Y_{1}$, respectively, therefore we can find two sequences $x_{0}^{n} \in X_{0}, x_{1}^{n} \in X_{1}, n \in \mathbb{Z}$, such that

$$
A x_{0}^{n}=A x_{1}^{n}=y_{n} \text { and }\left\|x_{0}^{n}\right\|_{X_{0}} \leq \gamma\left\|y_{n}\right\|_{Y_{0}}, \quad\left\|x_{1}^{n}\right\|_{X_{1}} \leq \gamma\left\|y_{n}\right\|_{Y_{1}} .
$$

Now we can define the required element $x \in\left(X_{0}, X_{1}\right)_{\theta, q}$ as

$$
x=\sum_{n} x_{1}^{n} \quad \text { for } \theta>\theta_{0},
$$

and

$$
x=\sum_{n} x_{0}^{n} \quad \text { for } \theta<\theta_{0} .
$$

Let us first consider the case of $\theta>\theta_{0}$. We note that if the series $x=\sum_{n} x_{1}^{n}$ converges in $X_{0}+X_{1}$ then we have $A x=\sum_{n} A x_{1}^{n}=\sum_{n} y_{n}=y$. To prove the convergence we need the inequality

$$
\left\|\sum_{n} x_{1}^{n}\right\|_{\left(X_{0}, X_{1}\right)_{\theta, q}} \leq \gamma\|y\|_{\left(Y_{0}, Y_{1}\right)_{\theta, q}} .
$$

As $A x_{0}^{n}=A x_{1}^{n}=y_{n}$, then $x_{0}^{n}-x_{1}^{n} \in \operatorname{Ker} A$ and hence $x_{0}^{n}-x_{1}^{n}=\lambda_{n} e$. Moreover, from $K\left(2^{k}, \lambda_{k} e ; \vec{X}\right) \approx\left|\lambda_{k}\right| 2^{k \theta_{0}}$ and (2) it follows that

$$
\left|\lambda_{k}\right| \leq \gamma 2^{-k \theta_{0}} K\left(2^{k}, \lambda_{k} e ; \vec{X}\right) \leq \gamma 2^{-k \theta_{0}}\left(\left\|x_{0}^{k}\right\|_{X_{0}}+2^{k}\left\|x_{1}^{k}\right\|_{X_{1}}\right) \leq \gamma 2^{-k \theta_{0}} J\left(2^{k}, y_{k} ; \vec{Y}\right) .
$$


(By $\gamma$ and $\gamma_{1}$ we will denote different positive constants in different contexts.) Hence

$$
\begin{aligned}
K\left(2^{n}, \sum_{k} x_{1}^{k} ; \vec{X}\right) & \leq K\left(2^{n}, \sum_{k<n} x_{0}^{k}+\sum_{k \geq n} x_{1}^{k} ; \vec{X}\right)+K\left(2^{n}, \sum_{k<n}-\lambda_{k} e ; \vec{X}\right) \\
& \leq\left\|\sum_{k<n} x_{0}^{k}\right\|_{X_{0}}+2^{n}\left\|\sum_{k \geq n} x_{1}^{k}\right\|_{X_{1}}+\sum_{k<n}\left|\lambda_{k}\right| K\left(2^{n}, e ; \vec{X}\right) \\
& \leq \sum_{k<n}\left\|x_{0}^{k}\right\|_{X_{0}}+2^{n} \sum_{k \geq n}\left\|x_{1}^{k}\right\|_{X_{1}}+\gamma 2^{\theta_{0} n} \sum_{k<n}\left|\lambda_{k}\right| \\
& \leq \gamma\left(\sum_{k} \min \left(1, \frac{2^{n}}{2^{k}}\right) J\left(2^{k}, y_{k} ; \vec{Y}\right)\right)+\gamma 2^{\theta_{0} n} \sum_{k<n} 2^{-k \theta_{0}} J\left(2^{k}, y_{k} ; \vec{Y}\right) .
\end{aligned}
$$

Therefore, the proof of the inequality (3) (and also the convergence of $\sum_{n} x_{1}^{n}$ in $X_{0}+X_{1}$ ) follows from (1) and the boundedness of the operators $S$ and $S_{\theta_{0}}$ in the space $l_{q}\left(\left\{2^{-n \theta}\right\}_{n \in \mathbb{Z}}\right)$. Here $S$ and $S_{\theta_{0}}$ are defined by the formulas

$$
\left(S\left\{a_{k}\right\}\right)_{n}=\sum_{k} \min \left(1, \frac{2^{n}}{2^{k}}\right) a_{k}, \quad\left(S_{\theta_{0}}\left\{a_{k}\right\}\right)_{n}=2^{\theta_{0} n} \sum_{k<n} 2^{-k \theta_{0}} a_{k}
$$

The boundedness of the first operator in the space $l_{q}\left(\left\{2^{-n \theta}\right\}_{n \in \mathbb{Z}}\right)$ follows from the fact that this operator is a discrete analog of the Calderón operator

$$
(S f)(t)=\int_{0}^{t} f(s) \frac{d s}{s}+t \int_{t}^{\infty} s^{-1} f(s) \frac{d s}{s},
$$

which is bounded in $L_{q}\left(t^{-\theta}, \frac{d t}{t}\right)$ for all $\theta \in(0,1)$.

The second operator $S_{\theta_{0}}$ is a discrete analog of the operator

$$
\left(S_{\theta_{0}} f\right)(t)=t^{\theta_{0}} \int_{0}^{t} s^{-\theta_{0}} f(s) \frac{d s}{s}
$$

which is bounded in $L_{q}\left(t^{-\theta}, \frac{d t}{t}\right)$ for $\theta>\theta_{0}$. Indeed, from the Minkovskii inequality 
we have

$$
\begin{aligned}
\left(\int_{0}^{\infty}\left(t^{-\theta}\left(S_{\theta_{0}} f\right)(t)\right)^{q} \frac{d t}{t}\right)^{\frac{1}{q}} & =\left(\int_{0}^{\infty}\left(t^{-\left(\theta-\theta_{0}\right)} \int_{0}^{t} s^{-\theta_{0}} f(s) \frac{d s}{s}\right)^{q} \frac{d t}{t}\right)^{\frac{1}{q}} \\
& =\left(\int_{0}^{\infty}\left(t^{-\left(\theta-\theta_{0}\right)} \int_{0}^{1}(t u)^{-\theta_{0}} f(t u) \frac{d u}{u}\right)^{q} \frac{d t}{t}\right)^{\frac{1}{q}} \\
& =\left(\int_{0}^{\infty}\left(t^{-\theta} \int_{0}^{1} u^{-\theta_{0}} f(t u) \frac{d u}{u}\right)^{q} \frac{d t}{t}\right)^{\frac{1}{q}} \\
& \leq \int_{0}^{1} u^{-\theta_{0}}\left(\int_{0}^{\infty}\left(t^{-\theta} f(t u)\right)^{q} \frac{d t}{t}\right)^{\frac{1}{q}} \frac{d u}{u} \\
& \leq \int_{0}^{1} u^{-\theta_{0}}\left(\int_{0}^{\infty}\left(\left(\frac{s}{u}\right)^{-\theta} f(s)\right)^{q} \frac{d s}{s}\right)^{\frac{1}{q}} \frac{d u}{u} \\
& \leq\left(\int_{0}^{\infty}\left(s^{-\theta} f(s)\right)^{q} \frac{d s}{s}\right)^{\frac{1}{q}} \cdot \int_{0}^{1} u^{\theta-\theta_{0}} \frac{d u}{u} \\
& \leq \gamma\left(\int_{0}^{\infty}\left(s^{-\theta} f(s)\right)^{q} \frac{d s}{s}\right)^{\frac{1}{q}} \cdot
\end{aligned}
$$

This concludes the proof for the case of $\theta>\theta_{0}$.

The case of $\theta<\theta_{0}$ can be considered in a similar way, we only need to define $x=\sum_{n} x_{0}^{n}$ and to prove that

$$
\left\|\sum_{n} x_{0}^{n}\right\|_{\left(X_{0}, X_{1}\right)_{\theta, q}} \leq \gamma\|y\|_{\left(Y_{0}, Y_{1}\right)_{\theta, q}} .
$$

This inequality is proved similarly to (3). We have

$$
\begin{aligned}
K\left(2^{n}, \sum_{k} x_{0}^{k} ; \vec{X}\right) \leq & K\left(2^{n}, \sum_{k<n} x_{0}^{k}+\sum_{k \geq n} x_{1}^{k} ; \vec{X}\right)+K\left(2^{n}, \sum_{k \geq n} \lambda_{k} e ; \vec{X}\right) \\
\leq & \left\|\sum_{k<n} x_{0}^{k}\right\|_{X_{0}}+2^{n}\left\|\sum_{k \geq n} x_{1}^{k}\right\|_{X_{1}}+\sum_{k \geq n}\left|\lambda_{k}\right| K\left(2^{n}, e ; \vec{X}\right) \\
\leq & \sum_{k<n}\left\|x_{0}^{k}\right\|_{X_{0}}+2^{n} \sum_{k \geq n}\left\|x_{1}^{k}\right\|_{X_{1}}+\gamma 2^{\theta_{0} n} \sum_{k \geq n}\left|\lambda_{k}\right| \\
\leq & \gamma\left(\sum_{k} \min \left(1, \frac{2^{n}}{2^{k}}\right) J\left(2^{k}, y_{k} ; \vec{Y}\right)\right) \\
& +\gamma 2^{\theta_{0} n} \sum_{k \geq n} 2^{-k \theta_{0}} J\left(2^{k}, y_{k} ; \vec{Y}\right) .
\end{aligned}
$$


Therefore, the inequality (3) follows from (1) and the boundedness of the operators $S$ (see (4)) and $S^{\theta_{0}}$ in $l_{p}\left(\left\{2^{-n \theta}\right\}_{n \in \mathbb{Z}}\right)$. Here $S^{\theta_{0}}$ is defined by the formula

$$
\left(S^{\theta_{0}}\left\{a_{k}\right\}\right)_{n}=2^{\theta_{0} n} \sum_{k \geq n} 2^{-k \theta_{0}} a_{k} .
$$

We already know that the operator $S$ is bounded in $l_{q}\left(\left\{2^{-n \theta}\right\}_{n \in \mathbb{Z}}\right)$ for all $\theta \in(0,1)$. The operator $S^{\theta_{0}}$ is a discrete analog of the operator

$$
\left(S^{\theta_{0}} f\right)(t)=t^{\theta_{0}} \int_{t}^{\infty} s^{-\theta_{0}} f(s) \frac{d s}{s}
$$

Its boundedness in $L_{q}\left(t^{-\theta}, \frac{d t}{t}\right)$ for $\theta<\theta_{0}$ follows from the Minkovskii inequality:

$$
\begin{aligned}
\left(\int_{0}^{\infty}\left(t^{-\theta}\left(S^{\theta_{0}} f\right)(t)\right)^{q} \frac{d t}{t}\right)^{\frac{1}{q}} & =\left(\int_{0}^{\infty}\left(t^{-\left(\theta-\theta_{0}\right)} \int_{t}^{\infty} s^{-\theta_{0}} f(s) \frac{d s}{s}\right)^{q} \frac{d t}{t}\right)^{\frac{1}{q}} \\
& =\left(\int_{0}^{\infty}\left(t^{-\left(\theta-\theta_{0}\right)} \int_{0}^{1}\left(\frac{t}{u}\right)^{-\theta_{0}} f\left(\frac{t}{u}\right) \frac{d u}{u}\right)^{q} \frac{d t}{t}\right)^{\frac{1}{q}} \\
& =\left(\int_{0}^{\infty}\left(t^{-\theta} \int_{0}^{1} u^{\theta_{0}} f\left(\frac{t}{u}\right) \frac{d u}{u}\right)^{q} \frac{d t}{t}\right)^{\frac{1}{q}} \\
& \leq \int_{0}^{1} u^{\theta_{0}}\left(\int_{0}^{\infty}\left(t^{-\theta} f\left(\frac{t}{u}\right)\right)^{q} \frac{d t}{t}\right)^{\frac{1}{q}} \frac{d u}{u} \\
& \leq \int_{0}^{1} u^{\theta_{0}}\left(\int_{0}^{\infty}\left((s u)^{-\theta} f(s)\right)^{q} \frac{d s}{s}\right)^{\frac{1}{q}} \frac{d u}{u} \\
& \leq\left(\int_{0}^{\infty}\left(s^{-\theta} f(s)\right)^{q} \frac{d s}{s}\right)^{\frac{1}{q}} \cdot \int_{0}^{1} u^{-\left(\theta-\theta_{0}\right)} \frac{d u}{u} \\
& \leq \gamma\left(\int_{0}^{\infty}\left(s^{-\theta} f(s)\right)^{q} \frac{d s}{s}\right)^{\frac{1}{q}} \cdot
\end{aligned}
$$

This completes the case of $\theta<\theta_{0}$, and it only remains to consider the case of $\theta=\theta_{0}$.

We need to show that the operator $A$ does not have an inverse on the space $\left(X_{0}, X_{1}\right)_{\theta_{0}, q}$. As the element $e \in \operatorname{Ker} A$ belongs to $\left(X_{0}, X_{1}\right)_{\theta_{0}, \infty}$, therefore $A$ does not have an inverse on $\left(X_{0}, X_{1}\right)_{\theta_{0}, \infty}$.

Let us consider the case of $\left(X_{0}, X_{1}\right)_{\theta_{0}, q}$ with $q<\infty$. In this case the kernel of $A$ does not intersect with $\left(X_{0}, X_{1}\right)_{\theta_{0}, q}$, but we will show that it is possible to construct a family of elements $x_{\varepsilon} \in\left(X_{0}, X_{1}\right)_{\theta_{0}, q}$ such that $\sup _{\varepsilon}\left\|A x_{\varepsilon}\right\|_{\left(Y_{0}, Y_{1}\right)_{\theta_{0}, q}}<\infty$ and $\lim _{\varepsilon \rightarrow 0}\left\|x_{\varepsilon}\right\|_{\left(X_{0}, X_{1}\right)_{\theta_{0}, q}}=\infty$. Hence the restriction of the operator $A$ on $\left(X_{0}, X_{1}\right)_{\theta_{0}, q}$ does not have an inverse.

To construct the family of elements $x_{\varepsilon} \in\left(X_{0}, X_{1}\right)_{\theta_{0}, q}$ we fix an arbitrary $\varepsilon \in(0,1)$ and consider the $K$-functional of the element $e$ on the three intervals $(0, \varepsilon],\left(\varepsilon, \varepsilon^{-1}\right)$, 
$\left[\varepsilon^{-1}, \infty\right)$. Let us denote by $\varphi_{0}^{\varepsilon}, \varphi_{1}^{\varepsilon}$, and $\varphi_{2}^{\varepsilon}$ the concave majorants of $K(\cdot, e ; \vec{X}) \chi_{(0, \varepsilon]}$, $K(\cdot, e ; \vec{X}) \chi_{\left(\varepsilon, \varepsilon^{-1}\right)}$, and $K(\cdot, e ; \vec{X}) \chi_{\left[\varepsilon^{-1}, \infty\right)}$ on $(0, \infty)$, i.e.,

$$
\begin{aligned}
\varphi_{0}^{\varepsilon} & =K(\cdot, e ; \vec{X}) \chi_{(0, \varepsilon)}+K(\varepsilon, e ; \vec{X}) \chi_{[\varepsilon, \infty)}, \\
\varphi_{1}^{\varepsilon} & =\frac{t}{\varepsilon} K(\varepsilon, e ; \vec{X}) \chi_{(0, \varepsilon]}+K(\cdot, e ; \vec{X}) \chi_{\left(\varepsilon, \varepsilon^{-1}\right)}+K\left(\varepsilon^{-1}, e ; \vec{X}\right) \chi_{\left[\varepsilon^{-1}, \infty\right)}, \\
\varphi_{2}^{\varepsilon} & =\frac{t}{\varepsilon^{-1}} K\left(\varepsilon^{-1}, e ; \vec{X}\right) \chi_{\left(0, \varepsilon^{-1}\right]}+K(\cdot, e ; \vec{X}) \chi_{\left(\varepsilon^{-1}, \infty\right)} .
\end{aligned}
$$

Then $K(\cdot, e ; \vec{X}) \leq \varphi_{0}^{\varepsilon}+\varphi_{1}^{\varepsilon}+\varphi_{2}^{\varepsilon}$ and from the $K$-divisibility theorem (see [3]) it follows that there exists a decomposition $e=x_{0}^{\varepsilon}+x_{1}^{\varepsilon}+x_{2}^{\varepsilon}$ such that

$$
K\left(\cdot, x_{i}^{\varepsilon} ; \vec{X}\right) \leq \gamma \varphi_{i}^{\varepsilon}, \quad i=0,1,2,
$$

with the constant $\gamma>0$ independent of $\varepsilon$. Let us take $x_{\varepsilon}=x_{1}^{\varepsilon}$. Then we only need to prove that

$$
\lim _{\varepsilon \rightarrow 0}\left\|x_{1}^{\varepsilon}\right\|_{\left(X_{0}, X_{1}\right)_{\theta}, q}=\infty
$$

and

$$
\sup _{\varepsilon}\left\|A x_{1}^{\varepsilon}\right\|_{\left(Y_{0}, Y_{1}\right)_{\theta_{0}, q}}<\infty .
$$

To prove (6) we note that from $K(t, e ; \vec{X}) \approx t^{\theta_{0}}$ and from the formulas (5) for $t \in\left[\varepsilon, \varepsilon^{-1}\right]$ it follows that

$$
K\left(t, x_{1}^{\varepsilon} ; \vec{X}\right) \geq K(t, e ; \vec{X})-K\left(t, x_{0}^{\varepsilon} ; \vec{X}\right)-K\left(t, x_{2}^{\varepsilon} ; \vec{X}\right) \geq \gamma t^{\theta_{0}}-\gamma_{1} \varepsilon^{\theta_{0}}-\gamma_{1} \frac{t}{\varepsilon^{-1}} \varepsilon^{-\theta_{0}} .
$$

Let us now fix a number $\delta \in(0,1)$. Then from the above inequality we have

$$
\lim _{\varepsilon \rightarrow 0}\left\|x_{1}^{\varepsilon}\right\|_{\left(X_{0}, X_{1}\right)_{\theta_{0}, q}} \geq\left(\int_{\delta}^{\delta^{-1}}\left(t^{-\theta_{0}} \gamma t^{\theta_{0}}\right)^{q} \frac{d t}{t}\right)^{\frac{1}{q}}=\gamma\left(2 \ln \frac{1}{\delta}\right)^{\frac{1}{q}} .
$$

Since $\delta \in(0,1)$ is arbitrary, we have $\lim _{\varepsilon \rightarrow 0}\left\|x_{1}^{\varepsilon}\right\|_{\left(X_{0}, X_{1}\right)_{\theta_{0}, q}}=\infty$. To prove $(7)$ it is sufficient to prove the following estimate

$$
K\left(t, A x_{1}^{\varepsilon} ; \vec{Y}\right) \leq \gamma \varepsilon^{\theta_{0}} \min \left(1, \frac{t}{\varepsilon}\right)+\gamma\left(\varepsilon^{-1}\right)^{\theta_{0}} \min \left(1, \frac{t}{\varepsilon^{-1}}\right) .
$$

The proof of (8) outside of the interval $\left[\varepsilon, \varepsilon^{-1}\right]$ follows from $K(t, e ; \vec{X}) \approx t^{\theta_{0}}$ and

$$
K\left(t, A x_{1}^{\varepsilon} ; \vec{Y}\right) \leq \gamma K\left(t, x_{1}^{\varepsilon} ; \vec{X}\right) \leq \gamma \varphi_{1}^{\varepsilon} \leq \gamma \frac{t}{\varepsilon} K(\varepsilon, e ; \vec{X}) \chi_{(0, \varepsilon]}+\gamma K\left(\varepsilon^{-1}, e ; \vec{X}\right) \chi_{\left[\varepsilon^{-1}, \infty\right)},
$$

and its proof inside the interval $\left[\varepsilon, \varepsilon^{-1}\right]$ follows from Lemma 1.2:

$$
\begin{aligned}
K\left(t, A x_{1}^{\varepsilon} ; \vec{Y}\right) & \approx \inf _{\lambda} K\left(t, x_{1}^{\varepsilon}-\lambda e ; \vec{X}\right) \leq K\left(t, x_{1}^{\varepsilon}-e ; \vec{X}\right) \leq K\left(t, x_{0}^{\varepsilon} ; \vec{X}\right)+K\left(t, x_{2}^{\varepsilon} ; \vec{X}\right) \\
& \leq \gamma K(\varepsilon, e ; \vec{X})+\gamma \frac{t}{\varepsilon^{-1}} K\left(\varepsilon^{-1}, e ; \vec{X}\right) .
\end{aligned}
$$

Thus the case of $\theta=\theta_{0}$ and the proof of Theorem A are complete. 


\section{References}

[1] S. V. Astashkin, On the interpolation of intersections by the real method, Algebra i Analiz 17 (2005), no. 2, 33-69 (Russian); English transl., St. Petersburg Math. J. 17 (2006), no. 2, 239-26.

[2] I. Asekritova and N. Kruglyak, On equivalence of $K$ - and J-methods for $(n+1)$-tuples of Banach spaces, Studia Math. 122 (1997), no. 2, 99-116.

[3] Yu. A. Brudnyı and N. Kruglyak, Interpolation functions and interpolation spaces, I, NorthHolland Mathematical Library, vol. 47, North-Holland Publishing Co., Amsterdam, 1991.

[4] J. Bergh and J. Löfström, Interpolation theory: An introduction, Grundlehren der Mathematischen Wissenschaften, vol. 223, Springer-Verlag, 1976.

[5] A. P. Calderón, Boundary value problems for the Laplace equation in Lipschitzian domains, Recent progress in Fourier analysis (El Escorial, 1983) (I. Peral and J. L. Rubio de Francia, eds.), North-Holland Math. Stud., vol. 111, North-Holland, Amsterdam, 1985, pp. 33-48.

[6] W. Cao and Y. Sagher, Stability of Fredholm properties on interpolation scales, Ark. Mat. 28 (1990), no. 2, 249-258.

[7] Stability in interpolation of Banach spaces, Proc. Amer. Math. Soc. 112 (1991), no. 1, $91-100$.

[8] G. David and S. Semmes, unpublished. For a formulation of the result see p. 127 of the paper by B. E. J. Dahlberg, C. E. Kenig, and G. C. Verchota, The Dirichlet Problem for the biharmonic equation in a Lipschitz domain, Ann. Inst. Fourier (Grenoble) 36 (1986), no. 3, 109-135.

[9] S. A. Ivanov and N. Kalton, Interpolation of subspaces and applications to exponential bases, Algebra i Analiz 13 (2001), no. 2, 93-115 (Russian); English transl., St. Petersburg Math. J. 13 (2002), no. 2, 221-239.

[10] N. Kalton and M. Mitrea, Stability results on interpolation scales of quasi-Banach spaces and applications, Trans. Amer. Math. Soc. 350 (1998), no. 10, 3903-3922.

[11] N. Kruglyak and M. Milman, A Distance between orbits that controls commutator estimates and invertibility of operators, Adv. Math. 182 (2004), no. 1, 78-123.

[12] N. Kruglyak, L. Maligranda, and L. E. Persson, On an elementary approach to the fractional Hardy inequality, Proc. Amer. Math. Soc. 128 (2000), no. 3, 727-734.

[13] (1999), no. 2, 323-344.

[14] J.-L. Lions and E. Magenes, Non-homogenous boundary value problems and applications, I, Grundlehren der mathematischen Wissenschaften, vol. 181, Springer-Verlag, New York, 1972.

[15] J. Löfström, Real interpolation with constraints, J. Approx. Theory 82 (1995), no. 1, 30-53.

[16] I. Ya. Shneiberg, On the solvability of linear operators in interpolation families of Banach spaces, Soviet Math. Dokl. 14 (1973), 1328-1331.

[17]_ Spectral properties of linear operators in interpolation families of Banach spaces, Mat. Issled. 9 (1974), no. 2 (32), 214-229, 254-255 (Russian).

[18] P. Sunehag, Subcouples of Codimension one and interpolation of operators that almost agree, J. Approx. Theory 130 (2004), no. 1, 78-98.

[19] A. Tabacco Vignati and M. Vignati, Spectral theory and complex interpolation, J. Funct. Anal. 80 (1998), no. 2, 383-397.

[20] M. Zafran, Spectral theory and interpolation of operators, J. Funct. Anal. 36 (1980), no. 2, $185-204$. 